\title{
Trastornos mentales y calidad de vida relacionada con la salud en profesionales de Unidades de Cuidados Intensivos
}

\author{
Mental disorders and health-related quality of life
}

in Intensive Care Units professionals

\author{
Higina Kelly Lemos Ferraz', Carolina Franco de Azevedo², \\ Juliet Valdelamar-Jiménez ${ }^{3}$, Carolina Villa Nova Aguiar ${ }^{4}$, \\ Marta Silva Menezes ${ }^{5}$ y Liliane Lins-Kusterer ${ }^{6}$
}

\begin{abstract}
${ }^{1}$ Especialista en Terapia Intensiva. Candidata a Maestría, Programa de Posgrado en Salud, Ambiente y Trabajo, Facultad de Medicina de Bahía, Universidad Federal de Bahía, Salvador, Bahía, Brasil. E-mail: higinakelly@hotmail.com

${ }^{2}$ Especialista en Salud de la Familia. Candidata a Maestría, Programa de Posgrado en Salud, Ambiente y Trabajo, Facultad de Medicina de Bahía, Universidad Federal de Bahía, Salvador, Bahía, Brasil. E-mail: cfakaroll@hotmail.com

${ }^{3}$ Magister en Medicina y Salud. Candidata a Doctorado, Programa de Posgrado en Medicina y Salud, Facultad de Medicina de Bahía, Universidad Federal de Bahía, Salvador, Bahía, Brasil. E-mail: jrvaldelamarj@unal.edu.co

${ }^{4}$ Doctora en Psicología. Profesora asistente, Escuela Bahiana de Medicina y Salud Pública, Salvador, Bahía, Brasil. E-mail: carol.vna@gmail.com

${ }^{5}$ Doctora en Medicina y Salud. Profesora titular, Escuela Bahiana de Medicina y Salud Pública, Salvador, Bahía, Brasil. E-mail: martasilvamenezes@gmail.com ${ }^{6}$ Doctora en Patología Humana. Posdoctora en Medicina y Salud Humana. Profesora de tiempo completo en la Facultad de Medicina de Bahía, Universidad Federal de Bahía, Salvador, Bahía, Brasil. E-mail: 1kusterer@gmail.com
\end{abstract}

El presente trabajo fue realizado con el apoyo de la Coordinación de Perfeccionamiento de Personal de Nivel Superior - Brasil (CAPES) - Código de financiación 001

Universidade Federal da Bahia, Largo do Terreiro de Jesus, n/s Centro Histórico, Salvador-BA, Brasil.

\section{Resumen}

Los profesionales de la salud de las Unidades de Cuidados Intensivos (UCI) enfrentan situaciones de sufrimiento humano, competitividad y demanda, que podrían perjudicar su calidad de vida y su salud mental. El objetivo del estudio fue describir los trastornos mentales comunes y la calidad de vida relacionada con la salud en profesionales de la salud de la UCI en Salvador, Brasil. Este estudio transversal con 195 profesionales utilizó un cuestionario sociodemográfico y laboral, el Self-Reporting Questionnaire (SRQ-20) de trastornos mentales comunes y el 36-Item Short Form Health Survey (SF-36v2) de calidad de vida relacionada con la salud. El $29.7 \%$ de los profesionales presentaron 
trastornos mentales comunes, especialmente entre profesionales de enfermería $(\mathrm{RP}=2.28$; IC $1.19-4.39 ; \mathrm{p}=.007)$. La calidad de vida relacionada con la salud para todos los profesionales estuvo disminuida, principalmente en función social $(44.25 \pm 10.15)$ y rol emocional $(45.86 \pm 10.58)$. El SRQ-20 correlacionó fuertemente con los dominios dolor corporal $(\mathrm{r}=$ $-.502)$, salud general $(\mathrm{r}=-.526)$, vitalidad $(\mathrm{r}=$ -.656), función social $(\mathrm{r}=-.608)$, salud mental $(\mathrm{r}=-.631)$ y el componente de salud mental $(\mathrm{r}=-.638)$ del SF-36v2 $(\mathrm{p}<.01)$. Los profesionales con trastornos mentales comunes mostraron una calidad de vida relacionada con la salud más deteriorada, esencialmente en dominios del componente de salud mental, y refirieron también dolor corporal. Es necesario discutir e implementar estrategias de evaluación, prevención y promoción de la salud mental entre los profesionales de las UCI para que sean consideradas dentro de las políticas de salud laboral.

Palabras clave: Unidad de Cuidados Intensivos, profesionales de la salud, calidad de vida relacionada con la salud, trastornos mentales comunes, SRQ-20, SF-36v2.

\section{Abstract}

Health professionals working in Intensive Care Units face situations of human suffering, competitiveness, demands for skills and job insecurity, which may have negative consequences on their quality of life and mental health. This scenario could trigger common mental disorders and impaired health-related quality of life in Intensive Care Unit workers. This study aimed to describe common mental disorders and health-related quality of life in Intensive Care Unit professionals in Salvador, Brazil. A descriptive, cross-sectional study was performed, with 195 health professionals working in the Intensive Care Unit of a high complexity hospital in Salvador, Bahia, Brazil. The professionals had to be working for three or more months in the units or removed from the service when they were detected with common mental diseases or other pathology. A socio-demographic and occupational data questionnaire, and the Self-Reporting Questionnaire (SRQ-20), was applied to identify common mental disorders, and the 36-Item Short Form Health Survey (SF-36v2) was used to assess health-related quality of life. The socio-demographic and occupational data questionnaire included age, sex, race, educational status, marital status, profession, number of working hours, the number of jobs, monthly income, and chronical diseases. Data was collected between March and July of 2019 in four Intensive Care Units, using Brazilian Portuguese translated versions of the instruments. The groups of professionals with and without common mental disorders were compared according to socio-demographic and occupational data and health-related quality of life using the $U$ Mann-Whitney test, with p-values under .05. Composite reliability evaluated the internal consistency of the instruments, assuming values above .70 as satisfactory. Bivariate analysis with the Spearman correlation test between the SRQ-20 and the SF-36v2 considered strong correlation values equal to or greater than .50 and a p-value of less than .05. The sample was composed of 137 $(70.3 \%)$ women and $148(72.3 \%)$ professionals who had more than 40 hours per week working in the Intensive Care Unit. Common mental disorders were detected in 58 (29.7 $\%$ ) health professionals, with a higher prevalence ratio in Nursing professionals (PR = 2.28; CI 1.19-4.39; $\mathrm{p}=.007)$. The SRQ-20 and the SF-36v2 domains showed satisfactory internal consistency, with values of composite reliability above .70. The normalized scores of health-related quality of life showed low average values in all domains, especially in Social Function (44.25 \pm 10.15$)$ and Emotional Role (45.86 \pm 10.58$)$. The SRQ-20 showed strong and significant correlations with Bodily Pain $(\mathrm{r}=-.502)$, General Health $(\mathrm{r}=-.526)$, Vitality $(\mathrm{r}=-.656)$, Social Function $(r=-.608)$, Mental Health $(r=-.631)$ and 
Mental Component Summary $(\mathrm{r}=-.638)$ of the SF-36v2 $(\mathrm{p}<.01)$. Health professionals with symptoms of common mental disorders had a more impaired health-related quality of life than those without symptoms. Specifically, the most affected domains were Social Function (36.07 \pm 9.68), Emotional Role (40.62 \pm 10.54$)$, Mental Health (40.53 \pm 9.67$)$, Mental Component Summary (37.71 \pm 9.72) and Bodily Pain $(40.55 \pm 9.00)$. Health professionals of the Intensive Care Units with symptoms of common mental disorders showed the lower health-related quality of life domains, presenting body pain compared to professionals without symptoms. The prevalence of common mental disorder symptoms in this study was similar to those reported in the literature and these disorders were more prevalent in Nursing professionals. Intensive Care Unit professionals showed low health-related quality of life, but those who presented symptoms of common mental disorders had the lower health-related quality of life. The implementation of strategies to evaluate, prevent and promote the mental health of Intensive Care Unit workers is necessary. Supportive workplace health policies should consider the mental health care of these professionals.

Keywords: Intensive Care Unit, healthcare professionals, quality of life related to health, common mental disorders, SRQ-20, SF-36.

\section{Introducción}

Las Unidades de Cuidados Intensivos (UCI) son sectores que prestan cuidados a los individuos en condiciones que requieren asistencia médica intensiva debido a la gravedad o riesgo clínico (Ministério de Saúde do Brasil, 2017). Los profesionales de la salud que conviven en este escenario se enfrentan a situaciones de sufrimiento y muerte, a factores ambientales, a la complejidad de las relaciones humanas y de trabajo, a un elevado grado de exigencias tanto competitivas como de habilidades, y a la precarización de sus derechos laborales. Estos factores pueden traen consecuencias psico- lógicas negativas que conllevarían a padecer una enfermedad mental (Ceballos-Vásquez et al., 2015; S. Ferreira, Santos y Estrela, 2016).

Entre las patologías que se pueden relacionar con el trabajo de los profesionales de UCI están los Trastornos Mentales Comunes (TMC). Estos son trastornos no psicóticos, caracterizados por un conjunto de síntomas como insomnio, ansiedad, depresión, irritabilidad, dificultad para concentrarse, olvidos, fatiga y quejas somáticas (Goldberg D, 1992), que no se encuadran en una categoría única de la 10. ${ }^{a}$ Clasificación Internacional de Enfermedades (CIE-10) o en los Manuales de Diagnóstico y Estadística (DSM) de la Asociación de Psiquiatría Americana (APA) (Rodrigues, Rodrigues, Oliveira, Laudano y Sobrinho, 2014). Varios estudios han identificado diferentes prevalencias de TMC en profesionales de la salud y su asociación con el entorno y proceso de trabajo (Alves, Pedrosa, Coimbra, Miranzil y Hass, 2015; Filho y Araújo, 2015). Sin embargo, son pocos los estudios que se enfocan en profesionales de la salud que atienden UCI en Brasil (Barbosa et al., 2020; Nascimento, Barbosa, Santos, Martins Júnior y Sobrinho, 2019)

Así como el trabajo en UCI puede estar asociado a la manifestación de TMC, también lo puede estar con la calidad de vida relacionada con la salud, definida como la influencia del estado de salud en la percepción de bienestar (Ebrahim, 1995). Los profesionales médicos pueden presentar una alta tasa de enfermedades mentales y un deterioro de la calidad de vida relacionada con la salud asociadas con el trabajo (Liu, Wang y Zhao, 2015). Más específicamente, el personal que atiende las UCI puede reportar síntomas musculo-esqueléticos que interfieren con su calidad de vida relacionada con la salud, sobre todo en aspectos sociales y en el desempeño de sus labores (de Oliveira, da Silva, Sousa y Shimizu, 2019).

Considerando que se han producido solo algunas investigaciones sobre los TMC en profesionales de UCI, en quienes las 
características relacionadas con su trabajo pueden contribuir a un deterioro en su salud (Ceballos-Vásquez et al., 2015; Nascimento et al., 2019), el objetivo del presente estudio es describir los TMC y la calidad de vida relacionada con la salud en profesionales de una UCI de la ciudad de Salvador, Brasil.

\section{Metodología}

Se trata de un estudio transversal y descriptivo, realizado en cuatro UCI de adultos y niños de un hospital de alta complejidad de la ciudad de Salvador, Brasil, en el año 2019.

\section{Participantes del estudio}

Fueron considerados como criterios de inclusión los profesionales de salud que estuvieran en estos servicios por un tiempo igual o superior a tres meses o a aquellos que hayan sido retirados del servicio al ser detectados con TMC u otra patología -esto, con el fin de reducir sesgos de selección. Los profesionales con actividades que no fuesen asistenciales dentro de la UCI no fueron incluidos en el estudio. De 220 profesionales pertenecientes a estas UCI, 195 profesionales (88.6\%) aceptaron participar y completaron los cuestionarios; entre ellos, 43 de Medicina, 48 de Enfermería, 30 de Fisioterapia y 74 de Técnica de enfermería.

\section{Procedimiento}

Los datos fueron recogidos entre marzo y julio de 2019 en las cuatro unidades de UCI. Los profesionales fueron convocados por las investigadoras para participar en el estudio por medio del consentimiento informado $\mathrm{y}$ respondieron los cuestionarios de variables sociodemográficas y ocupacionales, el Self-Reporting Questionnaire (SRQ-20) sobre TMC, y el 36-Item Short Form Health Survey questionnaire (SF-36v2) de calidad de vida relacionada con la salud. Los profesionales respondieron los cuestionarios de forma independiente, aunque podían consultar a las investigadoras si existían dudas.

\section{Variables de estudio}

La variable dependiente fue el TMC, evaluado mediante el SRQ-20. Las variables independientes fueron: la calidad de vida relacionada con la salud, evaluada con el SF-36v2, el sexo, la raza, la existencia de relaciones estables, la calificación profesional, la jornada de trabajo en UCI, el número de vínculos laborales, el tipo de profesión, la presencia de enfermedades crónicas, la edad, el ingreso económico mensual y el tiempo de formación.

\section{Self-Reporting Questionnaire (SRQ-20)}

El SRQ-20 está compuesto por 20 preguntas con opciones de respuesta dicotómicas, que hacen referencia a la percepción de síntomas psiquiátricos en los últimos 30 días. Las respuestas afirmativas reciben el valor de 1, mientras que las negativas no tendrán algún valor. Una puntuación igual o superior a 7 (la puntuación máxima es 20) puede indicar la presencia de TMC (Gonçalves, Stein y Kapczinski, 2008). El SRQ-20 ha sido validado en Brasil (Mari y Williams, 1986) y ha mostrado una consistencia interna satisfactoria con valores de alfa de Cronbach por encima de .80 en diferentes grupos ocupacionales, especialmente entre trabajadores de la salud (Santos, Carvalho y de Araújo, 2016).

\section{6-Item Short Form Health Survey Questionnaire (SF-36V2)}

El SF-36v2 es un instrumento de 36 ítems que evalúa la calidad de vida relacionada con la salud por medio de ocho dominios. Los 36 ítems, excepto el ítem 2, contribuyen a los puntajes de cada dominio, los cuales pueden ser agrupados en los Componentes de Salud Física (CSF) y Componentes de Salud Mental (CSM). Los tres dominios de Función Física (FF), Rol Físico (RF) y Dolor corporal (DC) 
contribuyen principalmente al CSF; los dominios de Salud Mental (SM), Rol Emocional (RE) y Función Social (FS) contribuyen al CSM. Los dominios de Vitalidad (VT), Salud General (SG) y Función Social se correlacionan con ambos componentes (Lins-Kusterer et al., 2019; Saris-Baglama et al., 2010; Ware, 2000). El programa PROCoRE versión 1.3 (licencia Optuminsight Life Sciences, Inc. número QM025905) permite calcular la puntuación de cada dominio y de los dos componentes para obtener puntuaciones normalizadas. La normalización de estos puntajes permite la comparación entre dominios, mediante el ajuste a una media de 50 y una desviación estándar de 10 . Para este estudio, se utilizó la versión adaptada al portugués brasilero de Lins-Kusterer et al. (2019), que obtuvo valores de consistencia interna (por los métodos de alfa de Cronbach y confiabilidad compuesta) superiores a .60 y fue validada estructuralmente por análisis factorial confirmatorio para una población de pacientes con VIH. Este instrumento también ha mostrado parámetros psicométricos adecuados dentro de población filipina, y se concluye que es efectivo para evaluar el estado de salud (Castillo-Carandang et al., 2013).

\section{Análisis estadístico}

Los datos fueron analizados con el programa SPSS versión 21. Se utilizó estadística descriptiva para analizar variables categóricas, como sexo, raza y presencia de TMC (frecuencia y porcentajes), y variables continuas, como edad e ingreso económico mensual (medias y desviación típica). Los grupos con y sin TMC fueron comparados a partir de la prueba U de MannWhitney $(\mathrm{p}<.05)$, teniendo en cuenta que la muestra no tiene una distribución normal. Se calculó la confiabilidad compuesta de los dominios del SF-36v2 y del SRQ-20, teniendo en cuenta valores superiores 70 como satisfactorios (Peterson y Kim, 2013).

Se realizó un análisis bivariado para identificar correlaciones entre el SRQ-20 y el SF-36v2 a partir de la prueba de correlación de
Spearman, considerando valores de .30 a .49 como correlación moderada y valores superiores o iguales a .50 como alta correlación (Cohen, 1988). Para los análisis de correlación se estableció una significancia estadística inferior al .05 .

\section{Aspectos éticos}

El estudio fue evaluado por el Comité de Ética en Investigación con Seres Humanos y aprobado a través del protocolo N. ${ }^{\circ} 3657114$. Todos los participantes firmaron el consentimiento libre e informado, de acuerdo a lo establecido en la Resolución 466/12 del Consejo Nacional de Salud de Brasil.

\section{Resultados}

De los 195 profesionales de la salud que participaron en el estudio, la mayoría fue de sexo femenino $(70.3 \%)$, autodeclarados pardos o negros $(82.6 \%)$ y sin enfermedades crónicas $(82.1 \%)$. Los participantes tuvieron una edad promedio de 51.5 años (DT = 118.9), además de un tiempo de formación profesional de alrededor de 10.7 años (DT $=6.0$ ). En cuanto a las características ocupacionales, aunque 148 participantes cumplían menos de 40 horas semanales en la UCI, 141 tenían más de un vínculo laboral con otras instituciones. El porcentaje de trabajadores con formación técnica fue de $36 \%$, frente a $30.4 \%$ con pregrado y $33 \%$ con posgrado.

Por otro lado, por medio del instrumento SRQ-20 se identificaron 58 trabajadores (29.7\%) con TMC. Los grupos con y sin TMC, aunque fueron semejantes en sus características sociodemográficas y ocupacionales, al no encontrarse diferencias significativas entre ellos, se observó que ser profesional de Enfermería tenía más probabilidades de padecer TMC comparado con el equipo médico de referencia $(\mathrm{RP}=2.28$; IC $1.19-4.39 ; \mathrm{p}=$ .007). La tabla 1 resume estas características, tanto para la muestra total como para cada grupo con y sin TMC. 
Tabla 1

Características sociodemográficas y ocupacionales de 195 trabajadores de UCI, con y sin TMC, en un hospital público de Salvador, Brasil, 2019.

\begin{tabular}{|c|c|c|c|c|c|c|}
\hline $\begin{array}{c}\text { Características } \\
\text { sociodemográficas } \\
\text { y ocupacionales }\end{array}$ & Total & $\begin{array}{c}\text { Con TMC } \\
(\mathbf{n}=\mathbf{5 8})\end{array}$ & $\begin{array}{l}\text { Sin TMC } \\
(n=137)\end{array}$ & $\mathbf{R P}$ & $\begin{array}{c}\text { IC } \\
(95 \%)\end{array}$ & $\mathbf{p}$ \\
\hline \multicolumn{7}{|l|}{ Sexo, $N(\%)$} \\
\hline Masculino & $58(29.7)$ & $12(20.7)$ & $46(79.3)$ & $*$ & - & - \\
\hline Femenino & $137(70.3)$ & $46(33.6)$ & $91(66.4)$ & 1.62 & $.93-2.83$ & .071 \\
\hline \multicolumn{7}{|l|}{ Raza, N (\%) } \\
\hline Branca & $34(17.4)$ & $9(26.5)$ & $25(73.5)$ & $*$ & - & - \\
\hline Parda/Negra & $161(82.6)$ & $49(30.4)$ & $112(69.6)$ & 1.15 & $.62-2.11$ & .645 \\
\hline \multicolumn{7}{|l|}{ Relación Estable, N (\%) } \\
\hline Sí & $93(47.7)$ & $28(30.1)$ & $65(69.9)$ & $*$ & - & - \\
\hline No & $102(52.3)$ & $30(29.4)$ & $72(76.6)$ & .98 & $.63-1.50$ & .915 \\
\hline \multicolumn{7}{|l|}{$\begin{array}{c}\text { Calificación profesional } \\
\qquad(N=194) \%\end{array}$} \\
\hline Técnico & $71(36.6)$ & $16(22.5)$ & $55(77.5)$ & $*$ & - & - \\
\hline Nivel superior o Posgrado & $123(63.4)$ & $41(33.3)$ & $82(66.7)$ & 1.47 & $.89-2.43$ & .111 \\
\hline \multicolumn{7}{|l|}{$\begin{array}{c}\text { Jornada de trabajo en } \\
\text { UCI, } N(\%)\end{array}$} \\
\hline Hasta 30 horas semanales & $52(27.1)$ & $13(25.0)$ & $39(75.0)$ & $*$ & - & - \\
\hline Más de 30 horas semanales & $140(72.9)$ & $44(31.4)$ & $96(68.6)$ & 1.26 & $.73-2.13$ & .386 \\
\hline \multicolumn{7}{|l|}{$\begin{array}{c}\text { Número de Vínculos } \\
\text { laborales, } N(\%)\end{array}$} \\
\hline Un vínculo & $54(27.7)$ & $17(31.5)$ & $37(68.5)$ & $*$ & - & - \\
\hline Más de un vínculo & $141(72.3)$ & $41(29.1)$ & $100(70.9)$ & .92 & $.57-1.47$ & .742 \\
\hline \multicolumn{7}{|l|}{ Tipo de profesión, $N(\%)$} \\
\hline Medicina & $43(22.1)$ & $9(20.9)$ & $34(79.1)$ & $*$ & - & - \\
\hline Fisioterapia & $30(15.4)$ & $9(30.0)$ & $21(70.0)$ & 1.43 & $.64-3.18$ & .376 \\
\hline Técnico de enfermería & $74(37.9)$ & $17(23.0)$ & $57(77.0)$ & 1.14 & $.56-2.33$ & .710 \\
\hline Enfermería & $48(24.6)$ & $23(47.9)$ & $25(52.1)$ & 2.28 & $1.19-4.39$ & .007 \\
\hline \multicolumn{7}{|l|}{ Enfermedad crónica, $N$ (\%) } \\
\hline No & $160(82.1)$ & $47(29.4)$ & $113(70.6)$ & $*$ & - & - \\
\hline Sí & $35(17.9)$ & $11(31.4)$ & $24(68.6)$ & .93 & $.54-1.61$ & .809 \\
\hline Edad, M (DT) & $51.5 \pm 118.9$ & $36 \pm 5$ & $58 \pm 141$ & - & - & $.254^{* *}$ \\
\hline $\begin{array}{l}\text { Tiempo de formación en } \\
\text { años, M (DT) }\end{array}$ & $10.7 \pm 6.0$ & $10 \pm 5$ & $11 \pm 7$ & - & - & $.481^{* *}$ \\
\hline $\begin{array}{c}\dagger \text { Ingreso económico, } \\
\text { M (DT) }\end{array}$ & $\begin{array}{c}6611.7 \pm 3 \\
587.2\end{array}$ & $\begin{array}{c}6476.63 \pm 3 \\
713.80\end{array}$ & $\begin{array}{c}6928.83 \pm 3 \\
277.64\end{array}$ & - & - & $.356^{* *}$ \\
\hline
\end{tabular}

*Categorías de referencia; **Prueba U de Mann-Whitney, $\mathrm{p}<.05 ; \dagger$ Real Brasilero. 
La confiabilidad compuesta para cada uno de los dominios del SF-36v2 presentó valores por encima de .70, sugiriendo una consistencia interna satisfactoria para esta muestra. En cuanto a la evaluación de calidad de vida relacionada con la salud, se encontraron puntuaciones promedio inferiores a la media de 50 en cada dominio, de acuerdo con la normalización del SF-36v2, y fueron Función Social (44.25 \pm 10.15$)$ y Rol Emocional $(45.86 \pm 10.58)$ los que obtuvieron promedios más bajos (Tabla 2). Por otro lado, el SRQ-20 también mostró una confiabilidad compuesta adecuada y un promedio de $4.99(\mathrm{DT}=4.10)$ en las puntuaciones de la muestra (Tabla 2).

Al evaluar las asociaciones entre el SF-36v2 y el SRQ-20, se observaron correlaciones negativas fuertes por encima de .50 con los dominios de Dolor Corporal, Salud General, Vitalidad, Función Social, Salud Mental y el Componente de Salud Mental, y correlaciones moderadas con Función Física, Rol Físico, Rol Emocional y el Componente de Salud Física $(p<.01)$ (Tabla 3).

Tabla 2

Confiabilidad compuesta y puntuaciones promedio de los dominios del SF-36v2 y del SRQ-20 en 195 trabajadores de UCI en un hospital público en Salvador, Brasil, 2019.

\begin{tabular}{c|c|c} 
SF-36v2 & $\begin{array}{c}\text { Confiabilidad } \\
\text { compuesta }\end{array}$ & M (DT) \\
\hline Función Física & .86 & $51.85 \pm 7.29$ \\
Rol Físico & .78 & $47.09 \pm 8.81$ \\
Dolor Corporal & .72 & $46.26 \pm 9.63$ \\
Salud General & .76 & $51.54 \pm 9.36$ \\
Vitalidad & .77 & $49.26 \pm 9.60$ \\
Función Social & .83 & $44.25 \pm 10.15$ \\
Rol Emocional & .76 & $45.86 \pm 10.58$ \\
Salud Mental & .77 & $49.44 \pm 10.00$ \\
Componente de Salud Física & - & $49.89 \pm 7.38$ \\
Componente de Salud Mental & - & $46.49 \pm 10.50$ \\
SRQ-20 & .84 & $4.99 \pm 4.10$
\end{tabular}

Tabla 3

Coeficientes de correlación de Spearman entre los dominios del SF-36v2 y el SRQ-20, en 195 trabajadores de UCI de un hospital público en Salvador, Brasil, 2019.

\begin{tabular}{c|c} 
Dominios del SF-36v2 & SRQ-20 \\
\hline Función Física & $-.405^{* *}$ \\
Rol Físico & $-.402^{* *}$ \\
Dolor corporal & $-.502^{* *}$ \\
Salud General & $-.526^{* *}$ \\
Vitalidad & $-.656^{* *}$ \\
Función Social & $-.608^{* *}$
\end{tabular}




\begin{tabular}{c|c} 
Dominios del SF-36v2 & SRQ-20 \\
\hline Rol Emocional & $-.466^{* *}$ \\
Salud Mental & $-.631^{* *}$ \\
Componente de Salud Física & $-.411^{* *}$ \\
Componente de Salud Mental & $-.638^{* *}$
\end{tabular}

**Correlación de Spearman, $\mathrm{p}<.01$ (bilateral).

Los trabajadores con TMC mostraron una calidad de vida relacionada con la salud mucho más disminuida, en comparación con aquellos que no tuvieron TMC. Las puntuaciones de los trabajadores con TMC estu- vieron por debajo de la media de normalización, con un importante compromiso en los dominios de Dolor Corporal, Función Social, Rol Emocional, Salud Mental y el Componente de Salud Mental.

Tabla 4

Puntuaciones de calidad de vida relacionada con la salud en 195 trabajadores de UCI, con y sin TMC, en un hospital público en Salvador, Brasil, 2019.

\begin{tabular}{c|c|c|c} 
& $\begin{array}{c}\text { Con TMC (n=58) } \\
\text { M (DT) }\end{array}$ & $\begin{array}{c}\text { Sin TMC (n=137) } \\
\text { M (DT) }\end{array}$ & p* \\
\hline Función Física & $48.66 \pm 8.53$ & $53.29 \pm 6.26$ & $<.001$ \\
Role Físico & $42.98 \pm 9.21$ & $48.83 \pm 8.06$ & $<.001$ \\
Dolor corporal & $40.55 \pm 9.00$ & $48.67 \pm 8.86$ & $<.001$ \\
Salud General & $44.35 \pm 9.46$ & $54.57 \pm 7.49$ & $<.001$ \\
Vitalidad & $41.27 \pm 7.97$ & $52.64 \pm 8.13$ & $<.001$ \\
Función Social & $36.07 \pm 9.68$ & $47.71 \pm 8.20$ & $<.001$ \\
Rol Emocional & $40.62 \pm 10.54$ & $48.08 \pm 9.82$ & $<.001$ \\
Salud Mental & $40.53 \pm 9.67$ & $53.21 \pm 7.44$ & $<.001$ \\
Componente de & $46.30 \pm 8.11$ & $51.41 \pm 6.51$ & $<.001$ \\
Salud Física & $37.71 \pm 9.72$ & $50.20 \pm 8.43$ & $<.001$ \\
Componente de & & &
\end{tabular}

*Prueba U de Mann-Whitney, $\mathrm{p}<.05$.

\section{Discusión}

El presente estudio buscó describir los trastornos mentales comunes y la calidad de vida relacionada con la salud en el ámbito ocupacional de profesionales de las Unidades de Cuidados Intensivos en Salvador, Brasil. En esta investigación, se encontró un porcentaje considerable de profesionales de UCI con TMC, y fueron los profesionales de Enfer- mería los que presentaron mayor prevalencia de estos trastornos. Los profesionales de estas unidades manifestaron una disminución en la calidad de vida relacionada con la salud, especialmente en dominios de Función Social y Rol Emocional. Sin embargo, el compromiso en la calidad de vida es más evidente entre los profesionales identificados con TMC. En ellos, hay un mayor deterioro en aspectos sociales, emocionales y mentales, además de 
una mayor manifestación de dolor corporal y menor rendimiento en el trabajo, debido a su limitada salud física. El SRQ-20 tuvo una correlación negativa fuerte con los dominios Dolor Corporal, Salud General, Vitalidad, Salud Mental, Función Social y el Componente de Salud Mental del SF-36v2.

En primer lugar, el porcentaje de TMC identificado entre los profesionales evaluados corrobora otras investigaciones realizadas con enfermeras y médicos de UCI, en las que la presencia de TMC fue detectada en $24.6 \%$ y $27.7 \%$ de los profesionales, respectivamente (Barbosa et al., 2020; Nascimento et al., 2019). En contraste, el porcentaje en este estudio es superior a los encontrados en otras investigaciones con profesionales de salud de atención primaria (Faria, Klosinski, Rustick y Oliveira, 2018; Gelaye et al., 2012).

Aunque en este estudio no se observaron diferencias significativas en las características sociodemográficas y ocupacionales entre los grupos con y sin TMC, sí se encontró una mayor prevalencia de TMC entre los profesionales de Enfermería. Esto respalda otras investigaciones en las que se ha encontrado una asociación entre las altas exigencias al trabajo de las enfermeras y la aparición de trastornos psiquiátricos menores (Araújo, Aquino, Menezes, Santos y Aguiar, 2003; Kirchhof et al., 2009).

En el caso de los profesionales de Enfermería de UCI, se han identificado algunos factores psicosociales inadecuados en esta unidad, que pueden contribuir a la sobrecarga mental -como las demandas psicológicas de este trabajo (sensoriales, emocionales, cognitivas), el conflicto y la falta de claridad del papel desempeñado y la dificultad para conciliar la vida profesional con la vida personal (Ceballos-Vásquez et al., 2015). Los profesionales de Enfermería realizan actividades gerenciales como la coordinación de todo el equipo de enfermería, administran las unidades y brindan atención al paciente crítico, lo cual requiere una alta carga física y mental que puede conducir a la ocurrencia de trastornos psicológicos (Nascimento et al., 2019).

En segundo lugar, el deterioro en la calidad de vida relacionada con la salud de los profesionales concuerda con los resultados de otra investigación realizada con médicos de UCI en Aracajú, Brasil, en la que se mostró un compromiso en aspectos físicos, psicológicos, sociales y ambientales (Costa et al., 2017). Sin embargo, otro estudio mostró que profesionales de Enfermería que atendían una UCI pediátrica tenían una calidad de vida relacionada con la salud preservada, aunque con un compromiso en la calidad del sueño debido a una posible adaptación a esta labor y a un sentimiento de gratificación por trabajar con niños (Guerra, Oliveira, Terreri y Len, 2016).

De forma más específica, se resalta el deterioro en aspectos sociales y emocionales en toda la muestra. No obstante, esto es más evidente en el grupo de profesionales con $\mathrm{TMC}$, en los que la Función social y el Rol Emocional tuvieron las más bajas puntuaciones. Una característica del trabajo en UCI es la disposición del personal para jornadas nocturnas, fines de semana y días festivos, lo que puede dificultar la convivencia con familiares y amigos y, así, contribuir al aislamiento social (Guerra et al., 2016) y a una reducción de hábitos saludables, como actividades físicas y de ocio (Cheung y Yip, 2015; Sousa et al., 2019). Por otro lado, el trabajo en el campo de la salud, debido a su alto grado de estrés y una consecuente adaptación forzada a los estresores de esta área, puede generar síntomas físicos, psíquicos y cognitivos, que impactan la vida de los profesionales (Paschoalini, Oliveira, Frigério, Dias y Santos, 2008).

Otros aspectos de la calidad de vida relacionada con la salud comprometidos entre los profesionales con TMC fueron los de Salud Mental y Vitalidad. El dominio de Salud Mental destaca aspectos relacionados con cambios de comportamiento, bienestar psicológico, así como ansiedad y depresión (Ware, 2000). El proceso y otros aspectos asociados al trabajo precario pueden aumentar la susceptibilidad de estos profesionales a la 
enfermedad mental que, a menudo, resulta en una pérdida del sentido de su trabajo y se manifiesta en sentimientos de ansiedad, irritabilidad, angustia e insatisfacción y depresión (Ferreira, Medeiros y Carvalho, 2017).

La vitalidad, referida en el SF-36v2 como el vigor para el desarrollo de las actividades diarias y aspectos relacionados con el nivel de energía y fatiga (Ciconelli, 1999), puede ser afectada en el contexto de UCI, al asumir altas cargas y malas condiciones de trabajo, lo que resulta en fatiga física y mental (Ceballos-Vásquez et al., 2015; Seligmann-Silva, Bernardo, Maeno y Kato, 2010). La muestra de este estudio presentó una alta prevalencia de profesionales con más de un vínculo laboral y con más de 30 horas semanales de trabajo en UCI, lo cual podría afectar su vitalidad.

Más allá del compromiso del componente mental de la calidad de vida relacionada con la salud en estos profesionales con TMC, se destaca también el alto reporte de dolor corporal y el deterioro en el rol físico que interfiere con sus actividades diarias. Estudios en Brasil y en otros países han demostrado la relación entre el dolor y la aparición de TMC en trabajadores (de Oliveira et al., 2019; Smith, Wei, Zhao y Wang, 2004). El trabajo de los profesionales de las UCI es intenso, complejo, agotador, con una alta carga de trabajo, estrés y exposición a riesgos laborales que pueden derivar en la aparición de dolores agudos o crónicos, como los relacionados con las lesiones musculo-esqueléticas, además de dolores de cabeza resultantes de las altas exigencias en el trabajo (Miranda, Magalhães, Moretão, Stival y Ramos, 2012; Neves, Vieira, Cardia, Lucena y Da-Silva, 2018). En consecuencia, se ha descripto en la literatura que la sobrecarga física presente en el trabajo de la salud, por su entorno insalubre, puede resultar en enfermedad física y limitaciones en el desempeño de actividades por parte del individuo (Oler, De Jesus, Barboza y Domingos, 2005). La limitación física genera inapetencia y contribuye a la aparición de enfermedades mentales (Pessoa, Cardia y Santos, 2010).
Por último, las correlaciones negativas y fuertes entre el SRQ-20 y varios dominios del SF-36v2, especialmente los que conforman el componente de Salud Mental y el de Dolor Corporal, muestran una asociación entre la presencia de TMC y un deterioro en la calidad de vida relacionada con la salud, lo que puede concordar con estudios anteriores (Alves, 2013; Jansen et al., 2011; Rocha, Scarlotto y Pizzinato, 2013). En un estudio realizado con trabajadores del poder judicial en el sur de Brasil, donde hay unas características de precarización laboral y cargas considerables de trabajo que también pueden afectar la salud mental, se encontró que quienes referían peor calidad de vida en relaciones sociales, aspectos físicos y condiciones ambientales tenían de dos a cuatro veces la probabilidad de presentar TMC (Rocha et al., 2013). En Brasil, un estudio abordó la enfermedad psíquica de los trabajadores de UCI y reportó poco reconocimiento y apoyo en el trabajo, crisis ética entre sus valores, sobrecarga laboral, trabajo en el turno de noche con subsecuentes alteraciones del sueño, dificultades en la relación con el jefe, rigidez institucional y dificultad para afrontar la muerte como factores que conducen a la enfermedad laboral (Monteiro, de Oliveira, Ribeiro, Grisa y Agostini, 2012)

Este estudio tiene como limitaciones el corte transversal que dificulta la determinación de una relación causal entre los TMC y la calidad de vida relacionada con la salud, y comparte el mismo cuestionamiento realizado por Rocha et al. (2013). Otra limitación fue su desarrollo en un solo hospital en Bahía, lo cual podría generar algún sesgo. No obstante, este hospital se caracteriza por ser de gran tamaño, y presenta UCI de distintas especialidades que brindan atención a una gran audiencia de usuarios del Sistema Único de Salud (SUS). Finalmente, en el presente estudio no se analizó la presencia de TMC ni la calidad de vida relacionada con la salud por tipo de UCI, lo cual puede ser un abordaje para futuras investigaciones. 


\section{Conclusión}

Los resultados presentados muestran una alta frecuencia de TMC y disminución de la calidad de vida relacionada con la salud en la población estudiada, lo que evidencia su asociación por correlaciones inversas significativas. Los profesionales de UCI identificados con TMC son, principalmente, de Enfermería y con un deterioro mayor de su calidad de vida, especialmente en dimensiones relacionadas con la salud mental y con manifestaciones de dolor corporal e interferencia de su estado físico en sus actividades en UCI. Estos hallazgos pueden contribuir a nuevas investigaciones sobre las enfermedades profesionales y sus impactos en los trabajadores de UCI, lo cual posibilitaría discusiones sobre los puntos críticos que comprometen el proceso de trabajo en salud y serviría de base para la formulación y mejora de acciones de promoción de la salud psicosocial y prevención de estos padecimientos en este escenario.

\section{Referencias}

Alves, A. P. (2013). Transtornos mentais comuns e qualidade de vida entre profissionais de saúde em um hospital de ensino. Universidade Federal do Triângulo Mineiro, Uberaba (MG). Alves, A, Pedrosa, L., Coimbra, M., Miranzil, M. y Hass, V. (2015). Prevalência de transtornos mentais comuns entre profissionais de saúde. Revista de Enfermagem UERJ, 23(1), 64-69.

Araújo, T. M., Aquino, E., Menezes, G., Santos, O. y Aguiar, L. (2003). Aspectos psicossociais do trabalho e distúrbios psíquicos entre trabalhadoras de enfermagem. Revista de Salud Pública, 37(4)). https://doi.org/10.1590/ S0034-89102003000400006

Barbosa, G., Santos, C., Oliveira, E., Filho, D., Nascimento, M. y Sobrinho, C. (2020). Distúrbios psíquicos menores em médicos intensivistas de cinco capitais brasileiras. Brazilian Journal of Development, 6(11), 90295-90301. https://doi.org/10.34117/bjdv6n11-441

Castillo-Carandang, N. T., Sison, O. T., Grefal, M.
L., Sy, R. G., Alix, O. C., Llanes, E. J. B., ... Wee, H. L. (2013). A community-based validation study of the short-form 36 version 2 philippines (tagalog) in two cities in the philippines. PLoS ONE, 8(12). https://doi.org/10.1371/ journal.pone.0083794

Ceballos-Vásquez, P., Rolo-González, G., Hérnandez-Fernaud, E., Díaz-Cabrera, D., Paravic-Klijn, T. y Burgos-Moreno, M. (2015). Fatores psicossociais e carga mental de trabalho: uma realidade percebida pelos enfermeiros em Unidades de Terapia Intensiva. Revista Latino-Americana de Enfermagem, 23(2), 315-322. https://doi.org/10.1590/0104-1169.0044.2557

Cheung, T. y Yip, P. S. F. (2015). Depression, anxiety and symptoms of stress among Hong Kong nurses: A cross-sectional study. International Journal of Environmental Research and Public Health, 12(9), 11072-11100. https:// doi.org/10.3390/ijerph120911072

Ciconelli, R. (1999). Tradução para o português e validação do questionário genérico de avaliação de qualidade de vida "Medical Outcomes Study 36-Item Short-Form Health Survey (SF-36)". Revista Brasileira de Reumatologia, 39(3), 143. http://repositorio.unifesp. br/handle/11600/15360

Cohen, J. (1988). Statistical Power Analysis for the Behavioral Sciences (2nd ed.). Hillsdale, NJ: Lawrence Erlbaum Associates.

Costa, K., Silva, D., Porto, A., Pinheiro, F., Souza, G., Bonfim, N., ... Araújo, D. (2017). Qualidade de vida de médicos intensivistas: um estudo transversal. Revista de Atençao Saúde, São Caetano do Sul, 15(54). https://doi. org/10.13037/ras.vol15n54.4792

de Oliveira, B., da Silva, V., Sousa, L. y Shimizu, I. (2019). Musculoskeletal symptoms and quality of life among workers at an intensive care unit in Teresina, Piauí, Brazil. Revista Brasileira de Medicina Do Trabalho, 17(4), 567-572. https://doi.org/10.5327/Z1679443520190381

Ebrahim, S. (1995). Clinical and public health perspectives and applications of health-related quality of life measurement. Soc. Sci. Med (10), 1383-1394. HTTPS://DOI10.1016/02779536(95)00116-o. 
Faria, N., Klosinski, R., Rustick, G. y Oliveira, L. (2018). Mental health of public health workers in Bento Gonçalves, Rio Grande do Sul,Brazil. Revista Brasileira de Medicina Do Trabalho, 16(2), 145-157. https://doi.org/10.5327/ Z1679443520180196

Ferreira, D., Medeiros, S. y Carvalho, I. (2017). Sofrimento psíquico no trabalhador de enfermagem: uma revisão integrativa. Revista de Pesquisa: Cuidado é Fundamental Online, $9(1), \quad 253 . \quad \mathrm{https}: / /$ doi.org/10.9789/21755361.2017.v9i1.253-258

Ferreira, S., Santos, M. y Estrela, F. (2016). Nursing activities score e o cuidado em uma unidade de terapia intensiva. Archives Health \& Sciences, 23(1), 63-67. https://doi.org/10.17696/23183691.23.1.2016.400

Filho, A. y Araújo, T. (2015). Estresse ocupacional e saúde mental dos profissionais do centro de especialidades médicas de Aracajú. Trabalho, Educação e Saúde, 13(suppl 1), 177-199. https://doi.org/10.1590/1981-7746-sip00016

Gelaye B., Lemma S., Deyassa, N., Bahretibeb. Y., Tesfaye. S., Berhane, Y. y Williams, M. A. (2012). Prevalence and Correlates of Mental Distress Among Working Adults in Ethiopia. Clinical Practice y Epidemiology in Mental Health, 8(1), 126-133. https://doi. org/10.2174/1745017901208010126

Goldberg, D. H. P. (1992). Common mental disorders: a bio-social model. https//doi. org.10.1111/j.1600-0447.1994.tb05916.x

Gonçalves, D., Stein, A. y Kapczinski, F. (2008). Avaliação de desempenho do Self-Reporting Questionnaire como instrumento de rastreamento psiquiátrico: um estudo comparativo com o Structured Clinical Interview for DSM-IV-TR. Cad. Saúde Pública, 24(2), 380-390. https://doi.org/10.1590/S0102311X2008000200017

Guerra, P., Oliveira, N., Terreri, M. y Len, C. (2016). Sono, qualidade de vida e humor em profissionais de enfermagem em Unidades de Terapia Intensiva Infantil. Revista de Escuela de Enfermería USP, 50(2), 279-285. https:// doi.org/10.1590/S0080-623420160000200014

Jansen, K., Mondín, T., Ores, L., Souza, L.,
Konradt, C., Pinheiro, R. y da Sliva, R. (2011). Transtornos mentais comuns e qualidadede vida em jovens: uma amostra populacional de Pelotas, Rio Grande do Sul, Brasil. Cadernos de Saúde Pública, 27(3), 440-448. https://doi. org/10.1590/S0102-311X2011000300005

Kirchhof, L., Magnago, T., Camponogara, S., Har-Ter Griep, R., Tavares, J., Prestes, F., y Paes, G. (2009). Condições de trabalho e características sóciodemográficas relacionadas à presença de distúrbios psíquicos menores em trabalhadores de enfermagem, 18(2). https:// doi.org/10.1590/S0104-07072009000200003

Lins-Kusterer, L., Valdelamar, J., Aguiar, C. V. N., Menezes, M. S., Netto, E. M. y Brites, C. (2019). Validity and reliability of the 36-Item Short Form Health Survey questionnaire version 2 among people living with HIV in Brazil. The Brazilian Journal of Infectious Diseases, 23(5). https://doi.org/10.1016/j. bjid.2019.08.001

Liu, C., Wang, L. y Zhao, Q. (2015). Factors related to health-related quality of life among Chinese psychiatrists: Occupational stress and psychological capital. BMC Health Services Research, 15(1). https://doi.org/10.1186/ s12913-015-0677-7

Mari, J. y Williams, P. (1986). A validity study of a psychiatric screening questionnaire (SRQ20) in primary care in the city of São Paulo. Br J Psychiatry , 148, 23-26. https://doi. org/10.1192/bjp.148.1.23

Ministério de Saúde do Brasil. (2017). Portaria $n^{\circ}$ 895, de 31 de março de 2017. Diário Oficial da União.

Miranda, A., Magalhães, C., Moretão, I., Stival, M. y Ramos, L. (2012). Dor crônica em trabalhadores de enfermagem de uma unidade de terapia intensiva. Journal of Nursing and Health, 2(1), 50-62. https://periodicos.ufpel. edu.br/ojs2/index.php/enfermagem/article/ view/3454

Monteiro, J., de Oliveira, A., Ribeiro, C., Grisa, G. y Agostini, N. (2012). Adoecimento Psíquico de Trabalhadores de Unidades de Terapia Intensiva. Psicologia Ciência E Profissão, 33(2), 366-379. https://doi.org/10.1590/ 
S1414-98932013000200009

Nascimento, D., Barbosa, G., Santos, C., Martins Júnior, D. y Sobrinho, C. (2019). Prevalência de distúrbio psíquico menor e fatores associados em enfermeiros intensivistas. Revista Baiana de Enfermagem, 1 33, e28091. https:// doi.org/10.18471/rbe.v33.28091

Neves, A. I. A., Vieira, E. M. A., Cardia, M. C. G., Lucena, N. M. G. y Da-Silva, L. B. (2018). Sociodemographic and organizational factors associated with musculoskeletal symptoms among intensive care unit professionals. Revista Brasileira de Medicina Do Trabalho, 16(3), 263-269. https://doi.org/10.5327/ Z1679443520180240

Oler, F., De Jesus, A., Barboza, D. y Domingos, N. (2005). Qualidade de vida da equipe de enfermagem do centro cirúrgico Life quality of nursing team in the surgical center. Arq Ciênc Saúde, 12(2), 102-112.

Paschoalini, B., Oliveira, M., Frigério, M., Dias, A. y Santos, F. (2008). Efeitos cognitivos e emocionais do estresse ocupacional em profissionais de enfermagem. Acta Paul Enferm, 21(3), 487-492. https://doi.org/10.1590/ S0103-21002008000300017

Pessoa, J., Cardia, M. y Santos, M. (2010). Análise das limitações, estratégias e perspectivas dos trabalhadores com LER/DORT, participantes do grupo PROFIT-LER: um estudo de caso. Ciência y Saúde Coletiva, 15(3), 821-830. https://doi.org/10.1590/S141381232010000300025

Peterson, R. y Kim, Y. (2013). On the relationship between coefficient alpha and composite reliability. Journal of Applied Psychology, 98(1), 194-8. . https://doi.org/10.1037/a0030767

Rocha, K., Scarlotto, M. y Pizzinato, A. (2013). Trastornos mentales comunes y calidad de vida: un estudio de trabajadores del sector público. Apuntes de Psicología, 31(3), 283-290.

Rodrigues, E., Rodrigues, U., Oliveira, L., Laudano, R. y Sobrinho, C. (2014). Prevalencia de trastornos mentales comunes en trabajadores de enfermería en un hospital de Bahia. Revista Brasileira de Enfermagem, 67(2), 296-301. https://doi.org/10.5935/00347167.20140040

Santos, K., Carvalho, F. y de Araújo, T. (2016). Internal consistency of the self-reporting questionnaire-20 in occupational groups. Revista de Saude Publica, 50, 6. https://doi.org/10.1590/ S1518-8787.2016050006100

Saris-Baglama, R., Dewey, C., Chisholm, G., Plumb, E., King, J., Kosinski, M., ... Ware, Jr., J. (2010). QualityMetric Health OutcomesTM Scoring Software 4.0. Lincoln, RI: QualityMetric Incorporated.

Seligmann-Silva, E., Bernardo, M., Maeno, M. y Kato, M. (2010). O mundo contemporâneo do trabalho e a saúde mental do trabalhador. Revista Brasileira de Saúde Ocupacional, 35(122), 187-191. https://doi.org/10.1590/ S0303-76572010000200002

Smith, D., Wei, N., Zhao, L. y Wang, R. (2004). Musculoskeletal complaints and psychosocial risk factors among Chinese hospital nurses. Occupational Medicine, 54(8), 579-582. https://doi.org/10.1093/occmed/kqh117

Sousa, K., Lopes, D., Tracera, G., Abreu, A., Portela, L. y Zeitoune, R. (2019). Transtornos mentais comuns entre trabalhadores de enfermagem de um hospital psiquiátrico. Acta Paulista de Enfermagem, 32(1), 1-10. https:// doi.org/10.1590/1982-0194201900002

Ware, J. E. (2000). SF-36 health survey update. Spine, 25(24), 3130-3139. https://doi. org/10.1097/00007632-20001215000008

Recibido: 4 de julio de 2020 Aceptado: 27 de octubre de 2021 